\title{
Switching behavior of Stoner-like magnetic thin film elements
}

\author{
M. Bauer, J. Fassbender, and B. Hillebrands \\ Fachbereich Physik und Forschungsschwerpunkt Materialwissenschaften, Erwin-Schrödinger-Straße 56, Universität Kaiserslautern, 67663 \\ Kaiserslautern, Germany
}

\begin{abstract}
We report results of the switching properties of thin magnetic films described by Stoner-like magnetic blocks subject to ultrashort, laterally localized magnetic field pulses, obtained by numerical investigations. Perpendicularly magnetized films exhibit a magnetization reversal due to a 4 ps magnetic field pulse, which can be explained with a coherent rotation model. Although the magnetic field pulse is short compared to the ferromagnetic resonance precession time, the time needed for the relaxation of the magnetization to the equilibrium state is rather large. In addition we show numerical results for the magnetization reversal of an in-plane magnetized sample with uniaxial in-plane anisotropy.
\end{abstract}

Recent experiments by the Siegmann group [1,2] have shown the validity of the Landau-Lifschitz-Gilbert equation on the time scale of a few picoseconds. It was possible to reverse the magnetization state of perpendicularly magnetized $\mathrm{Co} / \mathrm{Pt}$ films by a $50 \mathrm{GeV}$ electron bunch of $2-6 \mathrm{ps}$ duration penetrating perpendicularly through the sample generating in the film plane a circular magnetic field pulse. Although they compared their experiments to numerical simulations they restricted themselves to explain the final

$\mathrm{t}[\mathrm{ps}]$

$\frac{\mathrm{M}}{\mathrm{M}}$

4

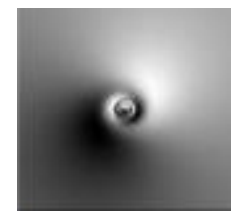

10

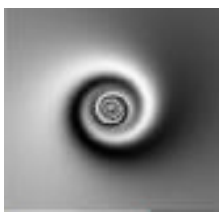

38

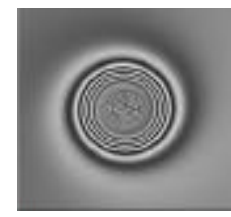

100

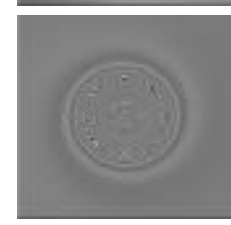

550
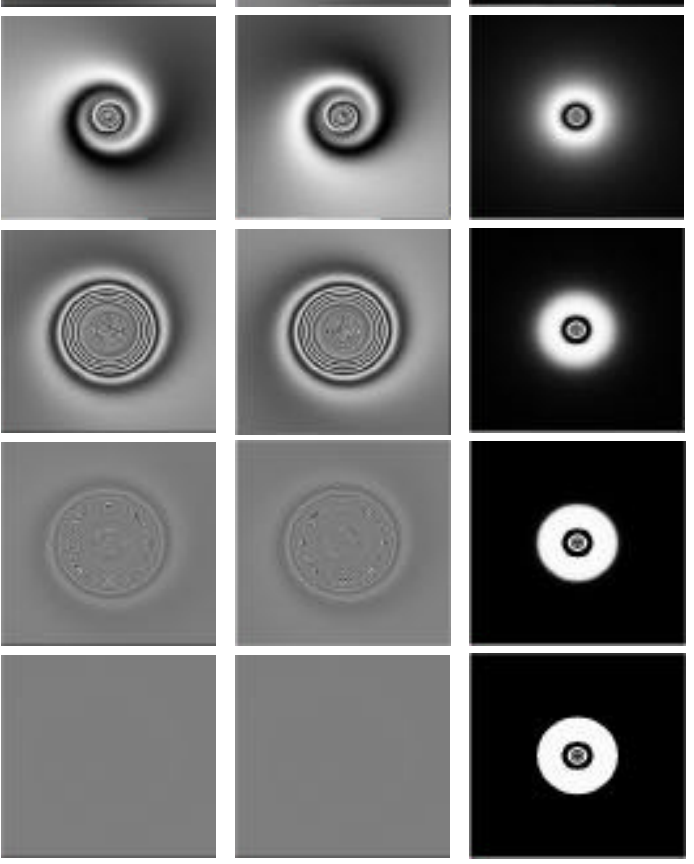

Figure 1: Three normalized mutually orthogonal magnetization components (left: $M_{x} / M_{S}$, middle: $M_{y} / M_{S}$, right: $M_{z} / M_{S}$ ) as a function of time (in ps) for a sample with a perpendicular anisotropy field of $50 \mathrm{kOe}$. The initial magnetization direction is along the $-z$-axis. The gray scale is a measure of the value of the magnetization component (black/white corresponds to -1/1). A magnetic field pulse of $4 \mathrm{ps}$ duration is applied at $\mathrm{t}=0 \mathrm{ps}$. Hence the first row indicates the situation just after the termination of the field pulse. A sample area of $100 \times 100 \mu \mathrm{m}^{2}$ is shown in all images. state of magnetization without commenting on the switching speed in different areas of the sample. In this paper we like to revisit the above mentioned experiment with special emphasis on the time evolution of the magnetization reversal.

We model the magnetic sample $\left(4 \pi \mathrm{M}_{\mathrm{S}}=17.8 \mathrm{kG}\right)$ by a twodimensional array of homogeneously magnetized, noninteracting thin film elements reflected by each pixel of an image. To compare our results to the experiments reported in $[1,2]$ we chose an electron pulse of 4 ps duration penetrating in the $-z$-direction through the center of the film ( $x y$-plane) creating a strong circular in-plane magnetic field. On this time scale the pulse shape plays no significant role, therefore we use a rectangular one. The magnetic field has a value of $100 \mathrm{kOe}$ at $\mathrm{r}=1 \mu \mathrm{m}$ apart from the center and varies with a $1 / \mathrm{r}$ dependence. The results are obtained by straightforward numerical integration of the Landau-Lifschitz equation with Gilbert damping term. For further details we refer to [3].

For a sample with a uniaxial perpendicular anisotropy field of $50 \mathrm{kOe}$ Fig. 1 shows the time evolution of all three mutually orthogonal magnetization components (left: $\mathrm{M}_{\mathrm{x}} / \mathrm{M}_{\mathrm{S}}$, middle: $\mathrm{M}_{\mathrm{y}} / \mathrm{M}_{\mathrm{S}}$, right: $\left.\mathrm{M}_{\mathrm{z}} / \mathrm{M}_{\mathrm{S}}\right)$ as a function of time. The gray scale reflects the value of the normalized magnetization components (-1/1 corresponds to black/white). Prior to the rectangularly shaped magnetic field pulse the sample is homogeneously magnetized along the $-z$-direction. Hence the $x$ - and $y$-component are gray in Fig. 1 and the $z$-component is black over the entire image (not shown in Fig. 1). The image size is $100 \times 100 \mu \mathrm{m}^{2}$. At the time $\mathrm{t}=0 \mathrm{ps}$ a bunch of electrons starts to penetrate perpendicularly through the center of the sample generating a circular in-plane magnetic field, which forces the magnetization vectors radially away from that center. The value of the in-plane magnetic field pulse is larger than that of the initial effective anisotropy field (sum of shape and perpendicular anisotropy field) within a radius of about $3 \mu \mathrm{m}$. Since the magnetization and the effective field direction are no longer colinear the magnetization starts to precess about the effective field direction. The area influenced by the in-plane magnetic field pulse is enlarged until the pulse terminates at the time $t=4$ ps. Hereafter, solely the effective anisotropy field, which is aligned along the $z$-direction, acts on the magnetization. Since $M_{z}$ varies across the sample, the effective anisotropy field and hence the precession frequency do so as well. A dephasing of neighboring magnetization vectors is the result (cf. $\mathrm{t}=10$, 
36 ps in Fig. 1). The areas where $M_{z}$ is \pm 1 at the pulse termination time the relaxation is completed immediately while for the areas where $\mathrm{M}_{\mathrm{z}} \approx 0$ the relaxation time is largest. The switching speed depends on (i) the angle between the actual and the equilibrium magnetization direction and (ii) the smaller the angle is the larger is $\mathrm{M}_{\mathrm{z}}$ and thus the precession frequency. During the relaxation of the magnetization $M_{z}$ increases, hence the precession frequency increases as well and the relaxation process accelerates further. We find the largest magnetization reversal time, indicated by the largest values of the $\mathrm{M}_{\mathrm{x}}-$ and $\mathrm{M}_{\mathrm{y}}$-components, at the boundaries between switched and non-switched areas (see $t=100 \mathrm{ps}$ in Fig. 1). The final magnetization state is reached in the whole sample after $\mathrm{t} \approx 550 \mathrm{ps}$. If the initial magnetization direction is $+z$ instead of $-z$, the magnetization vectors are forced radial to the center during the magnetic field pulse and the precession direction is inverted. The magnetization relaxation process itself is the same.

Figure 2 shows the much more complex behavior of a film with an in-plane uniaxial anisotropy field of $1 \mathrm{kOe}$ instead of a perpendicular one. The main difference is that in this case the in-plane anisotropy field and the shape anisotropy field are perpendicular to each other. Prior to the magnetic field pulse the sample is homogeneously magnetized along the $-x$ direction. The magnetic field pulse is the same as in Fig. 1. The image size is $300 \times 300 \mu \mathrm{m}^{2}$. The influence of the magnetic field pulse on the magnetization vectors in the sample

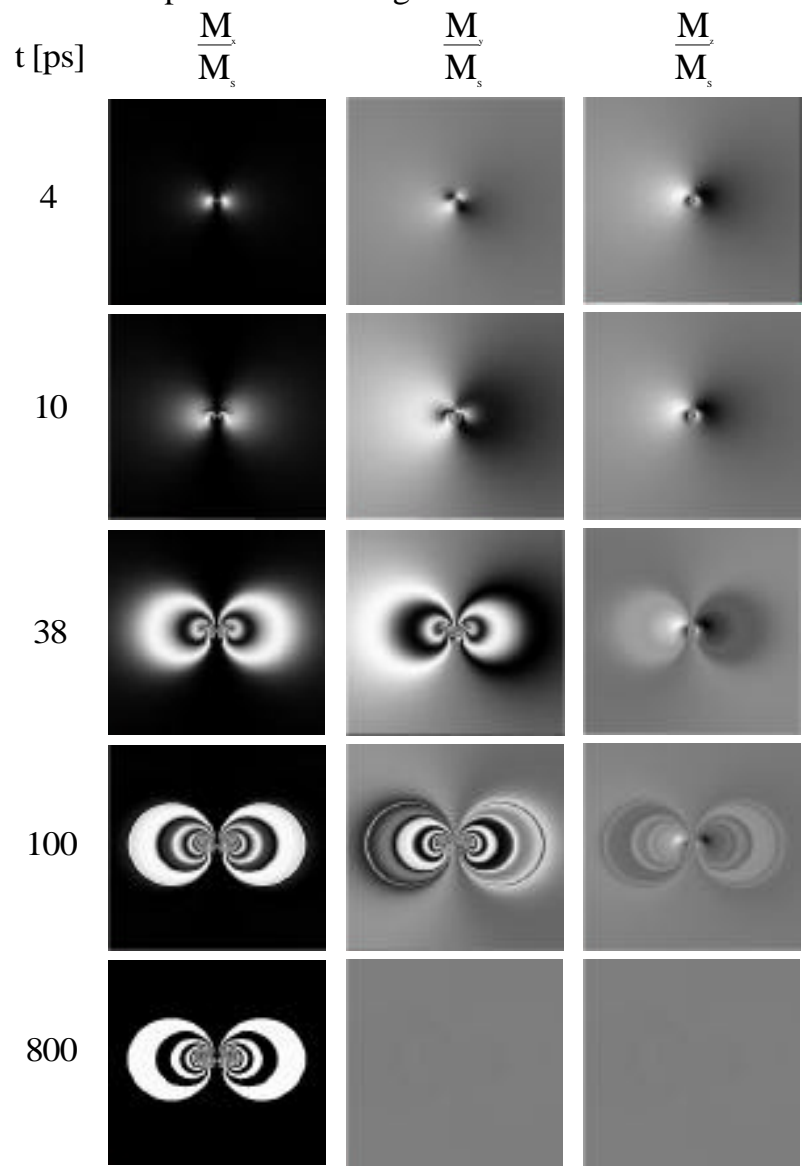

Figure 2: Three normalized mutually orthogonal magnetization components (left: $M_{x} / M_{S}$, middle: $M_{y} / M_{S}$, right: $M_{z} / M_{S}$ ) as a function of time (given in picoseconds) for a sample with a uniaxial in-plane anisotropy field of $1 \mathrm{kOe}$. The initial magnetization direction is along the $-x$-axis. A sample area of $300 \times 300 \mu \mathrm{m}^{2}$ is shown in all images. All other parameters are same as in Fig. 1. depends sensitively on their position due to two causes: i) the value of the magnetic field decreases with increasing distance from the center with a $1 / \mathrm{r}$-dependence and ii) the angle between $\vec{M}$ and $\vec{H}_{e x t}$ varies between $0^{\circ}-360^{\circ}$. Hence on the y-axis $(\mathrm{x}=0)$ the vector product of $\vec{M}$ and $\vec{H}_{e x t}$ is zero and the magnetization vectors remain unaffected. This leads to a symmetry with respect to the y-axis. At the first glance one would expect a symmetry with respect to the $\mathrm{x}$ axis as well, but the initial magnetization direction leads to a break in this symmetry. At the first moment of the field pulse the effective field has only in-plane components, and consequently the magnetization rotates towards the $\pm z$ direction leading to a strong demagnetizing field. The magnetization vectors start to precess about the effective field direction, which is no longer in-plane and changes continuously. At the time the pulse terminates $(t=4$ ps in Fig. 2) there are areas with large $z$-components of the magnetization near the center of the sample. In these areas the effective field has a large value (due to the shape anisotropy) and its direction is nearly perpendicular (out-of-plane). Further away from the center the effective field value decreases and its direction turns more and more into the film plane. Less energy is stored in these areas and hence the relaxation process proceeds faster than in the areas with the effective field nearly perpendicular to the film. This is clearly shown for $\mathrm{t}=100 \mathrm{ps}$ in Fig. 2, where in the latter mentioned areas the values of the $y$ - and z-magnetization components are large. Note that the contrast in the $z$-component is always weaker than in the $y$-component. This is a result of the large shape anisotropy field compared to the uniaxial in-plane anisotropy field. Hence it is much easier to rotate the magnetization vectors in the $y$-direction than in the $z$-direction. The perpendicular effective field leads to a rich variety of fine structure in the $x$ - and $y$-components of the magnetization (compare $\mathrm{t}=38 \mathrm{ps}, 100 \mathrm{ps}$ in Fig. 2) similar to the dephasing effect for the perpendicularly magnetized sample of Fig. 1. After about $\mathrm{t} \approx 800 \mathrm{ps}$ the $y$ - and $z$-components of the magnetization are relaxed in order to align the magnetization vectors parallel to the easy magnetization direction $( \pm x$-direction) in the final state.

The time evolution of the magnetization in thin magnetic films described by Stoner-like magnetic thin film elements subject to ultrashort, laterally localized magnetic field pulses has been discussed for two different anisotropies. For the final state of magnetization in the perpendicularly magnetized sample a good agreement with experiments is obtained. Although the magnetic field pulse is only 4 ps the time needed for the relaxation of the magnetization to the equilibrium position is of the order of several hundreds of ps.

We like to thank R. Lopusnik for critically reading of the manuscript. Support by Siemens AG, the german ministry of research and technology (BMBF), and the European Union (TMR project Dynaspin) is gratefully acknowledged.

\section{References}

[1] H.C. Siegmann, E.L. Garwin, C.Y. Prescott, J. Heidmann, D. Mauri, D. Weller, R. Allenspach, W. Weber, J. Magn. Magn. Mater 151, L8 (1995).

[2] C.H. Back, D. Weller, J. Heidmann, D. Mauri, D. Guarisco, E.L. Garwin, H.C. Siegmann, Phys. Rev. Lett. 81, 3251 (1998).

[3] M. Bauer, J. Fassbender, B. Hillebrands, R.L. Stamps, submitted to Phys. Rev. B. 